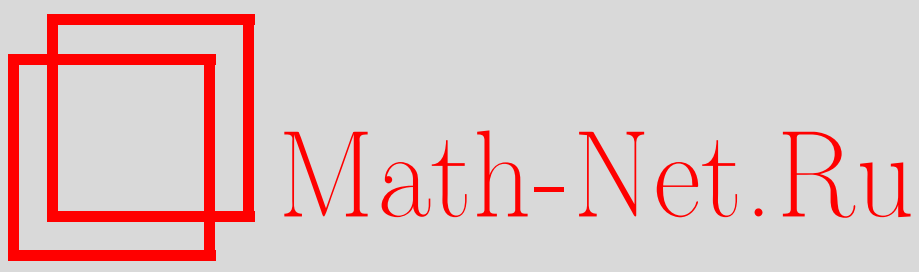

И. В. Протасов, Точки совместной непрерывности полугруппы ультрафильтров абелевой группы, Матем. сб., 1996, том 187, номер 2, 131-140

DOI: https://doi.org/10.4213/sm112

Использование Общероссийского математического портала Math-Net.Ru подразумевает, что вы прочитали и согласны с пользовательским соглашением http://www . mathnet.ru/rus/agreement

Параметры загрузки:

IP: 54.197 .130 .99

26 апреля 2023 г., 13:43:07 
УДК 512.536

\author{
И.В. Протасов
}

\title{
Точки совместной непрерывности полугруппы ультрафильтров абелевой группы
}

\begin{abstract}
Чех-стоунова компактификация $\beta G$ дискретной абелевой группы $G$ отождествляется с множеством всех ультраф̆илтров на группе $G$. Операция сложения на группе $G$ естественно продолжается до полугрупповой операции на $\beta G$. Пара улштрафильтров $(p, q)$ на группе $G$ является точкой совместной непрерывности полугруппы $\beta G$ тогда и только тогда, когда семейство подмножеств $\{P+Q: P \in p, Q \in q\}$ - база ультрафильтра. Основной результат статьи: если $G$ - счетная группа с конечным числом әлементов порядка $2, u$ $(p, q)$ - точка совместной непрерывности полугруппы $\beta G$, то по крайней мере один из ультрафильтров $p, q$ главный. При дополнителњных к $Z F C$ теоретико-множественных предположениях построены примеры, показывающие существенность ограничений на группу $G$.
\end{abstract}

Библиограффия: 5 названий.

Пусть $G$ - абелева группа, $\beta G$ - чех-стоунова компактификация группы $G$ как дискретного пространства. Элементами пространства $\beta G$ являются ультрафильтры на группе $G$, группа $G$ отождествляется с подмножеством всех главных ультрафильтров из $\beta G$. Ультраффильтры из подмножества $\beta G \backslash G$ называются свободныцми. Конструкция, возникшая в комбинаторике чисел, позволяет продолжить операцию сложения на группе $G$ до полугрупповой операции на $\beta G$. Операция сложения ультрафильтров оказывается непрерывной по второму аргументу при фиксированном первом аргументе, а также непрерьвной по первому аргументу, если фиксированный второй аргумент является главным ультраффильтром. Пару ультрафильтров $(p, q)$ назовем точкой совместной непрерьвности полугрупшы $\beta G$, если операция сложения ультрафильтров как отображение $\beta G \times \beta G \rightarrow \beta G$ непрерывна в точке $(p, q)$. Поскольку любой главный ультрафильтр является изолированной точкой пространства $\beta G$, то $(p, q)$ - точка совместной непрерывности, если по крайней мере один из ультрафильтров $p, q$ главный. Такие точки совместной непрерывности будем называть тривиальными. Естественно, для свободных ультрафильтров $p, q$ точка совместной непрерывности $(p, q)$ называется нетривиальной. Цель статьи - исследовать вопрос о сушествовании нетривиальных точек совместной непрерывности полугруппы $\beta G$.

К поиску таких точек приводит следуюшая задача. Пусть $p, q-$ ультрафильтры на группе $G$. Семейство подмножеств $\{P+Q: P \in p, Q \in q\}-$ база некоторого однозначно определенного фильтра $\varphi$ на группе $G$. Подмножество $A \subseteq G$ является элементом фильтра $\varphi$ тогда и только тогда, когда сушествуют такие подмножества $P \in p, Q \in q$, что $P+Q \subseteq A$. Сушествуют ли такие свободные ультрафильтры $p, q \in \beta G$, что фильтр $\varphi$ является ультрафильтром? Простые рассуждения, приведенные ниже в $\S 1$, показывают, что $\varphi$ - ультрафильтр тогда и только тогда, когда $(p, q)$ - точка совместной непрерывности полугруппы $\beta G$. 
Кратко о содержании статьи. Все рассматриваемые группы предполагаются абелевыми. В $\S 1$ изложена конструкция продолжения на $\beta G$ операции сложения элементов группы $G$ и приведены некоторые простые факты о точках совместной непрерывности. В частности, показано, что $(p, q)$ - точка совместной непрерывности полугруппы $\beta G$ для любых ультрафильтров $p, q$ из $\beta G$ тогда и только тогда, когда группа $G$ конечна.

В $\S 2$ доказано, что для счетной группы $G$ все точки совместной непрерывности полугруппы $\beta G$ тривиальны, если для любого свободного ультрафильтра $p \in \beta G$ уравнение $p+x=2 p+y$ неразрешимо в $\beta G$. Через $2 p$ обозначается ультрафильтр с базой $\{2 P: P \in p\}$, где $2 P=\{2 a: a \in P\}$.

Техника разбиений, развитая в $\S 3$, позволила установить неразрешимость в $\beta G$ уравнения $p+x=2 p+y$ для любого свободного ультрафильтра $p \in \beta G$ при условии, что подмножество всех элементов порядка 2 группы $G$ конечно. Доказано также, что для любой группы $G$ уравнение $x+x=2 x$ имеет в полугруппе $\beta G$ лишш тривиальные решения - главные ультрафильтры. Иначе говоря, $p+p \neq 2 p$ для любого свободного ультрафильтра $p$ на любой группе $G$.

Основные результаты статьи содержатся в $\S 4$. Если $G$ - счетная группа с конечным подмножеством элементов порядка 2 , то все точки совместной непрерьвности полугруппы $\beta G$ тривиальны. Примеры, построенные при дополнительных к асимптотике $Z F C$ теоретико-множественных предположениях (существование измеримых по Уламу кардиналов, аксиома Мартина), показывают, что отбросить ограничение счетности группы $G$ или конечности подмножества элементов порядка 2 , вообще говоря, нельзя. Отметим, что не известно ни одного "наивного" (т.е. построенного без дополнительных к $Z F C$ теоретико-множественных предположений) примера нетривиальной точки совместной непрерывности полугруппы $\beta G$ и сушествование таких примеров представляются автору весьма маловероятньм. Точнее, весьма правдоподобна гипотеза о существовании таких моделей системы аксиом $Z F C$, в которых все точки совместной непрерывности полугруппы $\beta G$ тривиальны для любой (или, по крайней мере, счетной) группы $G$. Без дополнительных предположений доказана тривиальность точек совместной непрерывности полугруппы $\beta G$ вида $(p, p)$ для любой группы $G$.

\section{§1. Конструкция продолжения и простейшие свойства точек совместной непрерывности}

Пусть $X$ - дискретное пространство, $\beta X$ - чех-стоунова компактификация пространства $X$. Элементами пространства $\beta X$ являются ультрафильтры на множестве $X$, а базу топологии образуют подмножества $\bar{A}=\{p \in \beta X: A \in p\}$, где $A$ пробегает все подмножества из $X$. При естественном отождествлении элементов из $X$ с главными ультрафильтрами подмножество $X$ плотно в $\beta X$. Отметим также, что пространство $\beta X$ экстремально несвязно, т.е. замыкание любого открытого подмножества из $\beta X$ открыто.

Следуюшее свойство пространства $\beta X$ является характеристичным: любое отображение $f$ пространства $X$ в компактное хаусдорфово пространство $K$ продолжается до непрерывного отображения $\bar{f}: \beta X \rightarrow K$. Если в качестве $K$ взять пространство $\beta X$, то для ультрафильтра $q \in \beta X$ базу ультрафильтра $\bar{f}(q)$ обра- 
зуют подмножества

$$
\bigcup\left\{F_{x}: x \in Q, F_{x} \in f(x)\right\},
$$

где $Q$ пробегает элементы ультрафильтра $q$, а $F_{x}$ - элементы ультрафильтра $f(x)$.

Предположим теперь, что $G$ - группа с дискретной топологией, и изложим конструкцию продолжения на $\beta G$ операции сложения элементов групшы $G$. Такая конструкция возникла и интенсивно используется в комбинаторике чисел (см., например, обзор [1]).

Для каждого элемента $a \in G$ определим отображение $R_{a}: G \rightarrow G$ правилом $R_{a}(x)=x+a$ для всех $x \in G$. Так как $G \subseteq \beta G$, то отображение $R_{a}$ продолжается до непрерывного отображения $\bar{R}_{a}: \beta G \rightarrow \beta G$. Ясно, что для каждого ультрафильтра $p \in \beta G \bar{R}_{a}(p)$ - ультрафильтр с базой $\{P+a: P \in p\}$. Таким образом, мы определили сумму $p+a=\bar{R}_{a}(p)$ ультрафильтра $p \in \beta G$ и элемента $a \in G$. Далее, для каждого ультрафильтра $p \in \beta G$ рассмотрим отображение $L_{p}: G \rightarrow \beta G$, заданное правилом $L_{p}(x)=p+x$ для всех $x \in G$. Продолжим отображение $L_{p}$ до непрерывного отображения $\bar{L}_{p}: \beta G \rightarrow \beta G$. Если $q \in \beta G$, то ультрафильтр $\bar{L}_{p}(q)$ называется суммой ультрафильтров $p$ и $q$ и обозначается $p+q$. Необходимые нам свойства непрерывности операции сложения ультрафильтров отмечены во введении.

Приведем следующее конструктивное описание ультрафильтра $p+q$. Возьмем произвольное подмножество $Q \in q$, и для каждого элемента $a \in Q$ выберем подмножество $P_{a} \in p$. Подмножество $\left\{P_{a}+a: a \in Q\right\}$ является элементом ультрафильтра $p+q$, и каждый элемент ультрафильтра $p+q$ содержит подмножество такого вида.

1.1. ЛЕммА. Для ультрафильтров $p, q \in \beta G(p, q)$ - точка совместной непрерьвности полугруппь $\beta G$ тогда и только тогда, когда для любого подмножества $R \in p+q$ найдутся такие подмножества $P \in p, Q \in q$, что $P+Q \subseteq R$.

ДокаЗАТЕльство. Пусть $(p, q)$ - точка совместной непрерьвности, $R \in p+q$. Для окрестности $\bar{R}$ ультрафильтра $p+q$ выберем, пользуясь непрерывностью операции сложения в точке $(p, q)$, такие подмножества $P \in p, Q \in q$, что $\bar{P}+\bar{Q} \subseteq \bar{R}$. Очевидно, что $P+Q \subseteq R$.

Пусть $R$ - произвольное подмножество из $p+q$. Выберем такие подмножества $P \in p, Q \in q$, что $P+Q \subseteq R$. Из определения операции сложения ультрафильтров следует, что $\bar{P}+\bar{Q} \subseteq \bar{R}$. Значит, $(p, q)$ - точка совместной непрерьвности.

1.2. ЗАмечаниЕ. Пусть $p, q \in \beta G, \varphi$ - фильтр на группе $G$ с базой $\{P+Q$ : $P \in p, Q \in q\}$. Так как $\varphi \subseteq p+q$, то по лемме $1.1 \varphi$-ультрафильтр тогда и только тогда, когда $(p, q)$ - точка совместной непрерывности полугруппы $\beta G$.

1.3. ЛЕмма. Если $(p, q)$ - точка совместной непрерывности полугруппь $\beta G$, mo $p+q=q+p$.

ДокАЗАТЕЛьСтво. Поскольку $q+p$ - ультрафильтр, достаточно показать, что $R \in q+p$ для любого подмножества $R \in p+q$. По лемме 1.1 подмножество $R$ содержит подмножество $P+Q$ для некоторых подмножеств $P \in p, Q \in q$. Так как $Q+P \in q+p$ и $P+Q=Q+P$, то $R \in q+p$. 
1.4. ЗАмЕчАниЕ. Хорошо известно (см., например, [2]), что полугруппа $\beta G$ некоммутативна для любой бесконечной группы $G$. Следовательно, по лемме 1.3 $(p, q)$ - точка совместной непрерьвности полугруппы $\beta G$ для любых ультрафильтров $p, q \in \beta G$ в том и только том случае, когда группа $G$ конечна.

\section{§ 2. Редукция к уравнениям}

2.1. ЛЕмма ФРОЛИКА [3, лемма 8.2]. Пусть $X$ - регулярное әкстремально несвязное пространство, $A, B$-счетные подмножества из $X, u \bar{A}, \bar{B}-и х$ замыкания в пространстве $X$. Если $\bar{A} \cap B=A \cap \bar{B}=\varnothing$, то $\bar{A} \cap \bar{B}=\varnothing$.

2.2. ЛЕмма. Пусть $G$ - счетная группа, $p, q, r, s \in \beta G$. Если $p+r=q+s$, то $p=q+t$, либо $q=p+h$ для некоторых ультрафильтров $t, h \in \beta G$.

ДоказАТЕльство. Так как $\beta G=\bar{G}$, то по условию леммы $(p+\bar{G}) \cap(q+\bar{G})$ $\neq \varnothing$. Из компактности пространства $\beta G$ и непрерывности операции сложения ультрафильтров по второму аргументу следует, что $p+\bar{G}, q+\bar{G}-$ замыкания в $\beta G$ подмножеств $p+G, q+G$. По лемме $2.1(p+\bar{G}) \cap(q+G) \neq \varnothing$, либо $(p+G) \cap(q+\bar{G}) \neq \varnothing$. Ввиду симметрии ограничимся первым случаем и выберем такие элементы $c \in \beta G, a \in G$, что $p+c=q+a$. Тогда $q=p+(c-a)$, где $c-a-$ ультрафильтр с базой $\{C-a: C \in c\}$. Положим $h=c-a$.

2.3. ЗАмЕЧАНИЕ. Можно показать, что условие счетности группы $G$ в лемме 2.2 существенно.

2.4. ЛЕмма. Если $G$ - счетная группа и полугруппа $\beta G$ имеет нетривиальную точку совместной непрерывности, то существует такой ультрафильтр $p \in \beta G \backslash G$, что уравнение $p+x=2 p+y$ разрешимо в полугруппе $\beta G$.

ДокАЗАТЕльство. Пусть $(p, q)$ - нетривиальная точка совместной непрерывности полугруппы $\beta G$. По лемме $1.3 p+q=q+p$. Ввиду леммы 2.2 можно считать, что $q=p+h$ для некоторого ультрафильтра $h \in \beta G$. Положим $r=p+q$ и покажем, что $r=2 p+h$. Фиксируем произвольное подмножество $R \in r$. Пользуясь леммой 1.1, выберем такие подмножества $P \in p, Q \in q$, что $P+Q \subseteq R$. Так как $q=p+h$ и $Q \in q$, то по непрерывности сложения по второму аргументу найдется такое подмножество $H \in h$, что $p+\bar{H} \subseteq \bar{Q}$. Возьмем произвольный элемент $a \in H$. Поскольку $p+a \in \bar{Q}$ и $a-$ главный ультрафильтр, то найдется такое подмножество $P_{a} \in p, P_{a} \subseteq P$, что $\bar{P}_{a}+a \subseteq \bar{Q}$. Так как $P_{a}+a \subseteq Q$ и $P_{a} \subseteq P$, то $P_{a}+P_{a}+a \subseteq R$. Заметим, что $2 P_{a}+a \subseteq P_{a}+P_{a}+a$, следовательно, $2 P_{a}+a \subseteq R$ для любого элемента $a \in H$. Поскольку $\left\{2 P_{a}+a: a \in H\right\}$ - элемент ультрафильтра $2 p+h$, то $R \in 2 p+h$. Ввиду произвола выбора подмножества $R \in r$ имеем $r=2 p+h$.

2.5. Лемма. Пусть $G, H$ - произвольнье группьи, $p \in \beta G, \bar{f}: \beta G \rightarrow \beta H$ - чех-стоуново продолжение гомоморфизма $f: G \rightarrow H$. Если уравнение $p+$ $x=2 p+y$ разрешимо в полугруппе $\beta G$, то уравнение $\bar{f}(p)+x=2 \bar{f}(p)+y$ разрешимо в полугруппе $\beta H$.

Доказательство непосредственно вытекает из того, что $\bar{f}$ - гомоморфизм соответствующих полугрупп, и соотношения $\bar{f}(2 p)=2 \bar{f}(p)$. 
2.6. ЛЕмма. Если $(p, p)$ - точка совместной непрерывности полугруппы $\beta G$, mо $p+p=2 p$.

ДоказАТеЛЬСтво. По лемме 1.1 семейство подмножеств $\{P+P: P \in p\}$ - база ультрафильтра $p+p$. Поскольку $P+P \in 2 p$ для любого подмножества $P \in p$, то $p+p=2 p$.

\section{§3. О разрешимости уравнения $p+x=2 p+y$ в полугруппе ультрафильтров}

3.1. Лемма. Пусть $\mathbb{Z}$ - группа челых чисел, $p \in \beta \mathbb{Z} \backslash \mathbb{Z}$. Уравнение $p+x=2 p+y$ неразрешимо в полугруппе $\beta \mathbb{Z}$.

ДоказАТельство. Допустим противное: $p+q=2 p+h$ для некоторых ультрафильтров $q, h \in \beta \mathbb{Z}$. Применяя лемму 2.5 к автоморфизму $f: G \rightarrow G$, заданному правилом $f(x)=-x$, можно считать, что множество натуральных чисел $\mathbb{N}$ является элементом ультрафильтра $p$.

Для натуральных чисел $a, b, a<b$, обозначим через $[a, b)$ полуинтервал числовой прямой $\{x \in \mathbb{R}: a \leqslant x<b\}$. Положим

$$
\begin{gathered}
B_{0}=[3,4), \quad B_{1}=[4,5), \quad B_{2}=[5,6), \quad B_{3}=[6,8), \quad B_{4}=[8,10), \quad B_{5}=[10,12), \\
A_{i}=\bigcup\left\{4^{n} B_{i} \cap \mathbb{N}: n<\omega\right\}, \quad i=0,1,2,3,4,5 .
\end{gathered}
$$

Легко проверяются следующие включения:

$$
2 A_{0} \subseteq A_{3}, \quad 2 A_{1} \subseteq A_{4}, \quad 2 A_{2} \subseteq A_{5}, \quad 2 A_{3} \subseteq A_{0}, \quad 2 A_{4} \subseteq A_{1}, \quad 2 A_{5} \subseteq A_{2}
$$

Поскольку $p$ - свободный ультрафильтр, $\mathbb{N} \in p$ и $\mathbb{N}=\{1,2\} \cup A_{0} \cup A_{1} \cup A_{2} \cup$ $A_{3} \cup A_{4} \cup A_{5}$, то найдется такой индекс $i$, что $A_{i} \in p$. Пусть для определенности $i=3$. В остальных случаях рассуждения аналогичны.

Возьмем произвольное целое число $b$. Ясно, что $a+b \in A_{2} \cup A_{3} \cup A_{4}$ для всех достаточно больших чисел $a \in A_{3}$. Так как $A_{3} \in p$, и $p$-свободный ультрафильтр, то $A_{2} \cup A_{3} \cup A_{4} \in p+b$. Значит, $p+G \subseteq \overline{A_{2} \cup A_{3} \cup A_{4}}$. Из непрерывности сложения по второму аргументу следует, что $p+\bar{G} \subseteq \overline{A_{2} \cup A_{3} \cup A_{4}}$. Следовательно, $p+q \in \overline{A_{2} \cup A_{3} \cup A_{4}}$, т.e. $A_{2} \cup A_{3} \cup A_{4} \in p+q$.

$\mathrm{C}$ другой стороны, $2 a+b \in A_{0} \cup A_{5} \cup A_{1}$ для всех достаточно больших чисел $a \in A_{3}$. Повторяя рассуждения из предыдушего абзаца, заключаем, что $A_{0} \cup A_{5} \cup A_{1} \in 2 p+h$.

Поскольку $\left(A_{2} \cup A_{3} \cup A_{4}\right) \cap\left(A_{0} \cup A_{5} \cup A_{1}\right)=\varnothing$, получаем противоречие с выбором ультрафильтров $p, q, h$.

3.2. ЛЕММА ДЕ БРЕЙНА-ЭРДЕША [3, лемма 3.1]. Пусть $X$ - произвольное множество, и отображение $f: X \rightarrow X$ не имеет неподвижных точек. $C y$ ществует такое разбиение $X=X_{1} \cup X_{2} \cup X_{3}$, что $f\left(X_{i}\right) \cap X_{i}=\varnothing, i=1,2,3$. 
3.3. ЛЕммА. Пусть $\alpha-$ предельный ординал, и группа $G$ представлена в виде обвединения $\bigcup\left\{G_{\gamma}: \gamma<\alpha\right\}$ возрастающей иепочки своих подгрупп $G_{\gamma}$. Предположим также, что $p \in \beta G \backslash G$, и для всех $\gamma<\alpha$ выполняются следующие условия:

1) факторгруппа $G_{\gamma+1} / G_{\gamma}$ не имеет әлементов порядка 2 ;

2) $G_{\gamma} \notin p$.

Уравнение $p+x=2 p+y$ неразрешимо в полугруппе $\beta G$.

ДоказАТЕльство. Допустим противное: $p+q=2 p+h$ для некоторых ультрафильтров $q, h \in \beta G$. Положим $X^{\gamma}=G_{\gamma+1} / G_{\gamma}$, и рассмотрим отображение $f_{\gamma}: X^{\gamma} \rightarrow X^{\gamma}$, заданное правилом $f_{\gamma}(x)=2 x$. Из условия 1 и леммы 3.2 вытекает существование такого разбиения множества $X^{\gamma} \backslash\{0\}=X_{1}^{\gamma} \cup X_{2}^{\gamma} \cup X_{3}^{\gamma}$, что $f_{\gamma}\left(X_{i}^{\gamma}\right) \cap X_{i}^{\gamma}=\varnothing, i=1,2,3$. Обозначим через $A_{i}^{\gamma}$ полный прообраз подмножества $X_{i}^{\gamma}$ при естественном гомоморфизме $G_{\gamma+1} \rightarrow G_{\gamma+1} / G_{\gamma}$. Тогда подмножества $A_{1}^{\gamma}$, $A_{2}^{\gamma}, A_{3}^{\gamma}$ попарно не пересекаются и вьполняются следующие соотношения:

$$
G_{\gamma+1} \backslash G_{\gamma}=A_{1}^{\gamma} \cup A_{2}^{\gamma} \cup A_{3}^{\gamma}, \quad A_{i}^{\gamma}+G_{\gamma}=A_{i}^{\gamma}, \quad 2 A_{i}^{\gamma} \cap A_{i}^{\gamma}=\varnothing, \quad i=1,2,3 .
$$

Положим $A_{i}=\bigcup\left\{A_{i}^{\gamma}: \gamma<\alpha\right\}, i=1,2,3$. Ясно, что подмножества $A_{1}, A_{2}, A_{3}$ попарно не пересекаются и выполняются следующие соотношения:

$$
G=G_{0} \cup A_{1} \cup A_{2} \cup A_{3}, \quad 2 A_{i} \cap A_{i}=\varnothing \quad i=1,2,3 .
$$

Так как $G_{0} \notin p$, то $A_{i} \in p$ для некоторого индекса $i$.

Покажем, что $A_{i} \in p+q$. Фиксируем произвольный элемент $a \in G$ и выберем такой индекс $\gamma$, что $a \in G_{\gamma}$. Поскольку $G_{\gamma} \notin p$, то $A_{i} \backslash G_{\gamma} \in p$. Из равенств $A_{i} \backslash G_{\gamma}=\bigcup\left\{A_{i}^{\beta}: \beta>\gamma\right\}, A_{i}^{\beta}+G_{\beta}=A_{i}^{\beta}, G_{\gamma} \subset G_{\beta}$ для всех $\beta>\gamma$, вытекает, что $\left(A_{i} \backslash G_{\gamma}\right)-a=A_{i} \backslash G_{\gamma}$. Следовательно, $A_{i} \backslash G_{\gamma} \in p+a$ и $A_{i} \in p+a$. В силу произвола выбора элемента $a \in G$ и соотношения $p+q \in p+\bar{G}$, заключаем, что $A_{i} \in p+q$.

Из условий 1), 2) следует, что $G_{\gamma} \notin 2 p$ для всех $\gamma<\alpha$. Так как $2 A_{i} \in 2 p$ и $2 A_{i} \cap A_{i}=\varnothing$, то найдется такой индекс $j \neq i$, что $A_{j} \in 2 p$. Рассуждения из предыдущего абзаца показьвают, что $A_{j} \in 2 p+h$.

Для фиксации противоречия с выбором ультрафильтров $q, h$ укажем на то, что $A_{i} \cap A_{j}=\varnothing$.

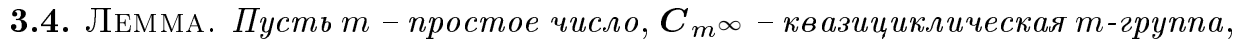
$p \in \beta \boldsymbol{C}_{m}^{\infty} \backslash \boldsymbol{C}_{m^{\infty}}$. Уравнение $p+x=2 p+y$ неразрешимо в полугруппе $\beta \boldsymbol{C}_{m} \infty$.

ДокАЗАТЕЛьство. Для $m \neq 2$ непосредственно применяем лемму 3.3. Поэтому предположим, что $p+q=2 p+h$ для некоторых ультрафильтров $q, h \in \beta \boldsymbol{C}_{2}$. Разобьем группу $\boldsymbol{C}_{2}$ на два подмножества $A_{0}, A_{1}$, где $A_{0}$ - совокупность всех элементов порядка $2^{n}, n$ - четное число, $A_{1}=G \backslash A_{0}, G=\boldsymbol{C}_{2}$. Выберем такой индекс $i$, что $A_{i} \in p$. Фиксируем произвольный элемент $a \in G$. Так как подмножество $\left(A_{i}-a\right) \backslash A_{i}$ конечно, а $p$ - свободный ультрафильтр, то $A_{i}-a \in p$ и $A_{i} \in p+a$. В силу произвольного выбора элемента $a$ получаем $A_{i} \in p+q$. С другой стороны, по определению разбиения $G \backslash A_{i} \in 2 p$, и аналогичные рассуждения приводят к тому, что $G \backslash A_{i} \in 2 p+h$. Наступило противоречие с выбором ультрафильтров $q, h$. 
3.5. Лемма. Пусть $G=\boldsymbol{C}_{2 \infty} \oplus \boldsymbol{C}_{3 \infty} \oplus \boldsymbol{C}_{5 \infty} \oplus \cdots, \oplus$ - знак прямой суммы, $p \in \beta G \backslash G$. Уравнение $p+x=2 p+y$ неразрешимо в полугруппе $\beta G$.

ДокАЗАТЕЛЬство. Введем следующие обозначения:

$$
G_{0}=C_{2^{\infty}}, \quad G_{1}=C_{2^{\infty}} \oplus C_{3 \infty}, \quad G_{2}=C_{2^{\infty}} \oplus C_{3^{\infty}} \oplus C_{5^{\infty}}, \ldots
$$

Тогда $G=\bigcup\left\{G_{n}: n<\omega\right\}$. Если $G_{n} \notin p$ для всех $n<\omega$, то применяем лемму 3.3. Поэтому можно считать, что $G_{m} \in p$ для некоторого $m<\omega$. Рассмотрим проектирование $f$ группы $G$ на прямое слагаемое $G_{m}$. Так как $\bar{f}(p)-$ свободный ультрафильтр на $G_{m}$, то по лемме 2.5 вопрос сводится к неразрешимости уравнения $\bar{f}(p)+x=2 \bar{f}(p)+y$ в полугрупше $\beta G_{m}$. В свою очередь, группа $G_{m}$ является прямой суммой конечного числа квазициклических слагаемых. Из конечности числа слагаемых следует, что проекция ультрафильтра $\bar{f}(p)$ по крайней мере на одно из этих слагаемых является свободным ультрафильтром. Повторное применение леммы 2.5 и леммы 3.4 завершает доказательство.

3.6. Лемма. Пусть $\mathbb{Q}$ - группа рациональных чисел, $p \in \beta \mathbb{Q} \backslash \mathbb{Q}$. Уравнение $p+x=2 p+y$ неразрешимо в полугруппе $\beta \mathbb{Q}$.

ДокАЗАТЕЛЬСТво. Предположим противное и рассмотрим группу $\mathbb{Z}$ как подгруппу групшы $\mathbb{Q}$. Если $f$ - факторный гомоморфизм группы $\mathbb{Q}$ на группу $\mathbb{Q} / \mathbb{Z}$, то по лемме 2.5 уравнение $\bar{f}(p)+x=2 \bar{f}(p)+y$ разрешимо в полугруппе $\beta(\mathbb{Q} / \mathbb{Z})$. Так как $\mathbb{Q} / \mathbb{Z} \simeq \boldsymbol{C}_{2} \oplus \boldsymbol{C}_{3} \infty \oplus \boldsymbol{C}_{5} \oplus \oplus \cdots$, то по лемме $3.6 \bar{f}(p)$-главный ультрафильтр. Представляя группу $\mathbb{Q}$ в виде объединения $\bigcup\left\{a_{n}+\mathbb{Z}: n<\omega\right\}$ смежных классов по подгруппе $\mathbb{Z}$, получаем, что $a_{m}+\mathbb{Z} \in p$ для некоторого $m<\omega$.

Уравнение $p+x=2 p+y$ запишем в виде

$$
\left(p-a_{m}\right)+x=2\left(p-a_{m}\right)+\left(a_{m}+y\right) .
$$

Из нашего предположения о разрешимости в $\beta \mathbb{Q}$ уравнения $p+x=2 p+y$ следует разрешимость уравнения $p_{1}+x=2 p_{1}+y$, где $p_{1}=p-a_{m}$. Заметим, что $\mathbb{Z} \in p_{1}$, и $p_{1}$ - свободный ультраффильтр. Выберем такие ультрафильтры $q, h \in \beta \mathbb{Q}$, что $p_{1}+q=2 p_{1}+h$. Применяя автоморфизм $f$ группы $\mathbb{Q}$, заданньй формулой $f(x)=-x$, можно считать, что множество натуральных чисел $\mathbb{N}$ является элементом ультрафильтра $p_{1}$.

Рассмотрим подмножества $A_{i}, i=0,1,2,3,4,5$, множества $\mathbb{N}$, определенные в ходе доказательства леммы 3.1. Положим $C_{i}=\bigcup\left\{A_{i}+a_{n}: n<\omega\right\}$. Одно из подмножеств $A_{i}$, скажем для определенности $A_{2}$, является элементом ультрафильтpa $p_{1}$.

Возьмем произвольный элемент $b \in \mathbb{Q}$. Представляя его в виде $b=c+a_{n}, c \in \mathbb{Z}$, замечаем, что $a+b \in C_{1} \cup C_{2} \cup C_{3}$ для всех достаточно больших чисел $a \in A_{2}$. Поэтому $C_{1} \cup C_{2} \cup C_{3} \in p_{1}+b$ и, в силу произвола выбора $b \in \mathbb{Q}, C_{1} \cup C_{2} \cup C_{3} \in p_{1}+q$.

$\mathrm{C}$ другой стороны, $2 a+b \in C_{4} \cup C_{5} \cup C_{0}$ для всех достаточно больших чисел $a \in A_{2}$. Следовательно, $C_{4} \cup C_{5} \cup C_{0} \in 2 p_{1}+h$. Наступило противоречие с тем, что $\left(C_{1} \cup C_{2} \cup C_{3}\right) \cap\left(C_{4} \cup C_{5} \cup C_{6}\right)=\varnothing$.

3.7. Теорема. Пусть $G$ - группа с конечным подмножеством әлементов порядка $2, p \in \beta G \backslash G$. Уравнение $p+x=2 p+y$ неразрешимо в полугруппе $\beta G$. 
ДокАЗАТЕЛЬСТво. Если указанное уравнение разрешимо в полугруппе $\beta G$, то по лемме 2.5 оно также разрешимо и в полугруппе $\beta D$, где $D$ - делимая оболочка группы $G$. Поскольку число элементов порядка 2 группы $D$ также конечно, можно считать группу $G$ делимой. Используя структурную теорему о делимых группах, представим группу $G$ в виде объединения $\bigcup\left\{G_{\gamma}: \gamma<\alpha\right\}$ возрастающей трансфинитной цепочки подгрупп $G_{\gamma}$, где $G_{0}$ - прямая сумма конечного числа квазициклических 2-подгрупп группы $G, G_{\gamma+1}=G_{\gamma} \oplus A_{\gamma}$, где $A_{\gamma}$ - квазициклическая группа либо группа рациональных чисел, и $G_{\gamma}=\bigcup\left\{G_{\beta}: \beta<\gamma\right\}$ для предельных ординалов $\gamma$.

Предположим вначале, что $G_{\gamma} \notin p$ для всех $\gamma<\alpha$, при этом автоматически $\alpha$ оказывается предельным ординалом. В этом случае доказательство завершается ссылкой на лемму 3.3 .

В альтернативном случае выберем наименьший ординал $\beta$, для которого $G_{\beta} \in p$. Проектируем группу $G$ на подгруппу $G_{\beta}$, которая является естественным прямым слагаемым группы $G$. Если $\beta$-предельньй ординал и $\beta>0$, то ввиду леммы 2.5 мы оказываемся в ситуации, рассмотренной в предыдущем абзаце. Иначе, имеется две возможности.

1) $\beta=0$. Так как $p$ - свободный ультрафильтр, $G_{0} \in p$ и $G_{0}$ - прямая сумма конечного числа квазициклических 2-подгруп, то проекция ультрафильтра $p$ по крайней мере на одно слагаемое группы $G_{0}$ оказывается свободньм ультрафильтром. Применяем леммы 2.5 и 3.4 .

2) $\beta=\gamma+1$ для некоторого ординала $\gamma$. Тогда $G_{\beta}=G_{\gamma} \oplus A_{\gamma}$. Из леммы 2.5 и лемм 3.4, 3.6 вытекает, что проекция ультрафильтра $p$ на слагаемое $A_{\gamma}$ является главным ультрафильтром. Поэтому $a+G_{\gamma} \in p$ для некоторого элемента $a \in A_{\gamma}$. Положим $p_{1}=p-a$ и заметим, что $\gamma<\beta$ и $G_{\gamma} \in p_{1}$. Разрешимость исходного уравнения равносильна разрешимости в полугруппе $\beta G$ уравнения $p_{1}+x=2 p_{1}+y$. Поскольку строго убываюшая цепочка ординалов конечна, доказательство теоремы завершено.

3.8. ЗАмЕчАниЕ. Условие конечности числа элементов порядка 2 в формулировке доказанной теоремы сушественно. Действительно, пусть $G$ - бесконечная группа экспоненты 2 . Возьмем произвольный ультрафильтр $p \in \beta G \backslash G$. Так как $2 p=0$, то уравнение $p+x=2 p+y$ имеет в полугруппе $\beta G$ очевидное решение $x=q, y=p+q$, где $q-$ произвольный ультрафильтр из $\beta G$.

3.9. Теорема. Если $G$ - произвольная группа, $p \in \beta G$ u $p+p=2 p$, mo $p-$ главный ультрафильтр.

ДокАЗАТЕЛЬство. Обозначим через $H$ подгруппу всех 2-элементов группы $G$, $f-$ факторный гомоморфизм групшы $G$ на $G / H$. Так как $\bar{f}(p)+\bar{f}(p)=2 \bar{f}(p)$, и группа $G / H$ не имеет элементов порядка 2 , то по теореме $3.7 \bar{f}(p)$ - главньй ультрафильтр. Следовательно, $H+a \in p$ для некоторого элемента $a \in G$. Заменяя ультрафильтр $p$ на $p-a$ и учитывая очевидное равенство $(p-a)+(p-a)=2(p-a)$, можно считать, что $H \in p$. Рассмотрим два случая.

1) Для любого подмножества $P \in p$ порядки элементов из $P$ не ограничены в совокупности. Разобьем подгруппу $H$ на два подмножества $A_{0}, A_{1}$, где $A_{0}$ - совокупность всех элементов порядка $2^{n}, n$ - четное число, $A_{1}=H \backslash A_{0}$. Так как $H \in p$, 
то $A_{i} \in p$ для некоторого $i=0,1$. По определению разбиения $H \backslash A_{i} \in 2 p$. Возьмем произвольный элемент $b \in H$ и выберем такое подмножество $P \in p, P \subseteq A_{i}$, что порядки всех элементов из $P$ больше порядка элемента $b$. Тогда $P+b \subseteq A_{i}$ и, значит, $A_{i} \in p+p$. Так как $\left(H \backslash A_{i}\right) \cap A_{i}=\varnothing$, то $p+p \neq 2 p$ и этот случай исключается.

2) Сушествует такое подмножество $P \in p, P \subseteq H$, что порядки всех элементов из $P$ ограничены в совокупности. Выберем натуральное число $n$ так, что $2^{n} a=0$ для всех элементов $a \in P$. Из равенства $p+p=2 p$ вытекает, что $p+\cdots+p=2^{n} p$, где число слагаемых в левой части равно $2^{n}$. Так как $2^{n} P=\{0\}$, то $2^{n} p$ - главный ультрафильтр. Поскольку свободные ультрафильтры образуют подполугруппу полугрупшы $\beta G$, то слагаемое $p$ из левой части является главньм ультрафильтром, и теорема доказана.

\section{§4. Точки совместной непрерывности}

4.1. ТеоремА. Если $G$ - счетная группа с конечныцм числом әлементов

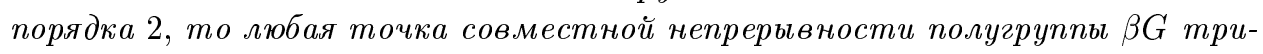
виальна.

Доказательство непосредственно следует из леммы 2.4 и теоремы 3.7.

4.2. ТЕОРемА. Для любой группы $G$ все точки совместной непрерывности полугруппы $\beta G$ вида $(p, p)$ и $(p,-p)$ тривиальньь.

ДоказАтЕльство. Для точек вида $(p, p)$ утверждение вытекает из леммы 2.6 и теоремы 3.9. Пусть $(p,-p)$ - точка совместной непрерывности полугруппы $\beta G$. Так как $-p=\{-P: P \in p\}$, то по лемме 1.1 любое подмножество группы $G$, являюшееся элементом ультрафильтра $p+(-p)$ содержит 0. Значит, ультрафильтр $p+(-p)$ главный. Поскольку свободные ультрафильтры образуют подполугруппу полугрупшы $\beta G$, то $p$-главный ультрафильтр.

4.3. ЗАмЕчанИЕ. Используя утверждение теоремы 4.2 , относящееся к точкам $(p, p)$, и теорему Рисса-Сушкевича для полугруппы ультрафильтров [4], можно доказать тривиальность точек совместной непрерывности $(p, q)$, где $p$ - ультрафильтр из минимального идеала полугруппы $\beta G$.

4.4. ПримеР. Предположим, что мощность группы $G$ измерима по Уламу. Укажем нетривиальную точку совместной непрерывности полугруппы $\beta G$. По одному из эквивалентных определений измеримости по Уламу на группе $G$ существует свободный счетно полньй ультрафильтр $p$. Фиксируем произвольньй свободный ультрафильтр $q$ на группе $G$, элементом которого является некоторое счетное подмножество из $G$. Возьмем произвольное подмножество $R \in p+q$, и выберем такие счетное подмножество $Q \in q$ и подмножества $P_{a} \in p, a \in Q$, что $\bigcup\left\{P_{a}+a: a \in Q\right\} \subseteq R$. Положим $P=\bigcap\left\{P_{a}: a \in Q\right\}$. Из счетной полноты ультрафильтра $p$ следует, что $P \in p$. Поскольку $P+Q \subseteq R$, по лемме $1.1(p, q)-$ точка совместной непрерывности полугруппы $\beta G$.

4.5. Вопрос. Пусть $G$ - группа без элементов порядка 2 и мощность групшы $G$ неизмерима по Уламу. Верно ли, что все точки совместной непрерьвности полугрупшы $\beta G$ тривиальны? 
4.6. ПримеР. Используя аксиому Мартина, Е. Г. Зеленюк в работе [5] построил на счетной группе $G$ экспоненты 2 такой фильтр $\varphi$ с базой из подгрупп, что подмножество $\{h \in \beta G: \varphi \subseteq h\}$ состоит из главного ультрафильтра 0 и таких свободных ультрафильтров - идемпотентов $p, q$, что $p+q=q+p=q$. Проведенный автором анализ этой конструкции показал, что $(p, q)$ - точка совместной непрерывности полугруппы $\beta G$.

4.7. Вопрос. Совместно ли с аксиоматикой $Z F C$ утверждение о том, что для счетной группы $G$ экспоненты 2 все точки совместной непрерывности полугруппы $\beta G$ тривиальны?

Положительный ответ на этот вопрос можно получить, если доказать следующую гипотезу. Если сушествует нетривиальная точка совместной непрерьвности полугруппы $\beta G$, то в пространстве $\beta \omega \backslash \omega$ имеется $P$-точка. Как известно, в некоторых моделях системы аксиом $Z F C$ пространство $\beta \omega \backslash \omega$ не имеет $P$-точек. Некоторое основание для формулировки гипотезы дает тот факт, что существование фильтра $\varphi$, описанного в примере 4.6 , влечет наличие $P$-точки в пространстве $\beta \omega \backslash \omega$.

\section{Список литературы}

1. Hindman N. Ultrafilters and combinatorial number theory // Lect. Notes Math. V. 751, 1979. P. 49-184.

2. Baker J. W., Milnes P. The ideal structure of the Stone-Čech compactification of group // Math. Proc. Camb. Phil. Soc. 1977. V. 82. P. 401-409.

3. Comfort $W$. $W$. Ultrafilters: some old and some new results // Bull. Amer. Math. Soc. 1977. V. 83. P. $417-454$.

4. Ruppert $W$. Compact semitopological semigroups: an intrinsic theory // Lect. Notes Math. V. 1079, 1984. P. 1-260.

5. Зеленюк Е. Г. Топологические групшы с конечньми полугруппами ультрафильтров // Докл. АН Украины. 1995. №6 (в печати).

Киевский университет им. Т. Шевченко

Поступила в редакцию 24.11 .1994 